\title{
Trajectory Tracking Fuzzy Control Algorithm for Picking Robot Arm
}

\author{
Zhang Zhiyong ${ }^{1}$, Zhang JianFeng ${ }^{1 *}$, Huang Lvwen ${ }^{l}$ and Li ChangYue \\ ${ }^{1}$ College of information Engineering, \\ Northwest A\&F University, Yangling, Shaanxi, 712100, China \\ Izzy@nwsuaf.edu.cn,zjf@nwsuaf.edu.cn
}

\begin{abstract}
Fuzzy tracking control discuss the pure model behavior and ignore the original mathematical model, make the picking robot arm gain accurate perception ability to complex environmental condition, control its velocity and reach smoothly the target position. This paper proposed fuzzy tracking controller algorithm for obtaining the joints position relative to the desired trajectory, which drive stably 3 joints tending to be stable in $1.5 \mathrm{~s}$ on the conditions of changeable environment, reflecting the strong robustness and stability of the tracking control system, enhancing their positioning and grasping stability to restrain picking robot arm chattering.
\end{abstract}

Keywords: Picking stability; Fuzzy control; Picking arm; Robotics

\section{Introduction}

The problem of picking stability with nonlinear dynamic equations to unknown environment is one of the most challenging problems. Zhang MingLu and Jiao Xinjing [1] applied the classical control theory to gain accurate mathematic model, have already produced substantial results. But intelligent control method based on the model of system behavior such as fuzzy control method, the ant colony algorithm etc is pay sustainable attention by Ahamed W [2].But so far these method is need to be improved further. Especially, fuzzy control just discussed the pure behavior model and ignored the original mathematical model, only discussed the offline stage fuzzy logic modeling system. In order to make the picking arm apperceive accurately complex environmental condition, to regulate their speed and reach smoothly the target position. The paper proposed fuzzy tracking controller algorithm on obtaining the desired joints tracking trajectory, so the good picking coordination ability and stable driving velocity and angle velocity were maintained on the conditions of changing environment, to enhance their positioning and grasping stability. Trajectory tracking fuzzy control should be combined with two times curve scheme. Firstly, the fuzzy controller regulate flexibly, not to establish accurate mathematical model, avoiding the complexity computation and uncertainty analysis, can be set to adjust the output control quantity by reference to Wang Hongq ${ }^{[1]}$ analysis, so as to ensure the smoothness and stability of control system, and it is essential to solve the large specific deviation problem according to the tracking algorithm.

As for the problem to rectifying deviation algorithms on tracking control for picking robot arm, Wu Xing [3] avoided the robot arm unstable movement phenomenon in the path tracking, using two sub-fuzzy controllers. Couceiro M [4] proposed a synchronous control algorithm in method of assumptions forecast adjusting iteration to ensure the elimination of picking robot arm programming path deviation of synchronous speed and stability. Aiming to problems on 
initial state position error is so great to result in instability system. Efe $M$ et.al [6] proposed a temporary solution on trajectory tracking. Firstly some temporary trajectory was planned which picking robot arm smoothly moved to the expecting point, then combined fuzzy control property of path tracking and picking arm movement performance, if the deviation of actual position was greater than the desired, the controller would not track trajectory and lose stability due to the influence of fuzzy saturation characteristic, so fuzzy control is suitable for the little deviation between posture and the desired trajectory, precise trajectory tracking is apt to be achieved. When outside disturbances deviation such as wind speed is great, quadratic curve tracking would be replaced fuzzy control till the deviation was eliminated and then switched back to the fuzzy control. Its control scheme is described as following, according to the current target trajectory and picking arm posture, the reference point is regarded as picking robot arm arriving at the expected position, then chose fuzzy tracking strategies based on the calculated deviation, when the deviation is greater than the given threshold value, the quadratic curve path tracking is adopt to rectify faster deviation, when the deviation is less than the given threshold value, fuzzy following strategy is adopt to follow a desired trajectory till the deviation decreased to conform to the conditions of fuzzy tracking strategies.

\section{Representation of Picking Robot Arm Trajectory Tracking}

The current posture shown as Fig.1 of picking robot arm is expressed as $(x, y, \theta)$, the expectation posture expressed as $(x, y, \theta)$, furthermore the input controlling point of picking robot arm is indicated as $P_{P_{r}}\left(v_{r}, w_{r}\right)$, trajectory error of picking arm can be described as following in the coordinates

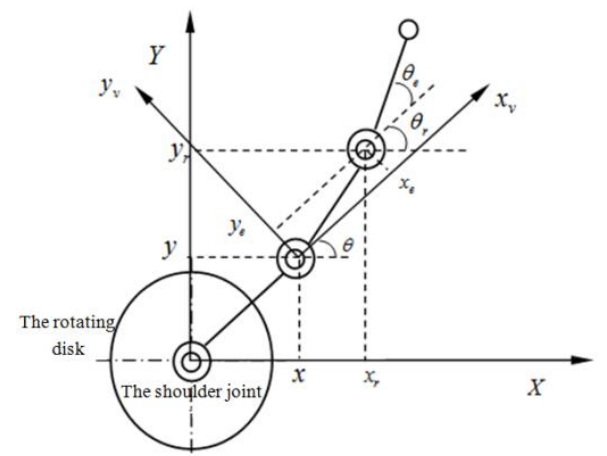

Figure.1 Diagram of Pose Error for Picking-arm in the Coordinate

$$
\begin{aligned}
& \left\lceil x_{e}\right\rceil\lceil\cos \theta \quad \sin \theta \quad 0\rceil\left\lceil x_{r} \quad-x\right\rceil \\
& \left|\begin{array}{l}
y_{e} \\
y_{e}
\end{array}\right|=\left|\begin{array}{ccc}
-\sin \theta & \cos \theta & 0
\end{array}\right|\left|\begin{array}{ll}
|| y_{r} & -y
\end{array}\right| \\
& \left\lfloor\theta_{e}\right\rfloor\left\lfloor\begin{array}{lll}
0 & 0 & 1
\end{array}\right\rfloor\left\lfloor\begin{array}{ll}
\theta_{r} & -\theta
\end{array}\right\rfloor
\end{aligned}
$$

The differential posture error equations can be described as following 


$$
\left\{\begin{array}{l}
x_{e}=w y_{e}-v+v_{r} \cos \theta_{e} \\
y_{e}=-w x_{e}+v_{r} \sin \theta_{e} \\
\theta_{e}=w_{r}-w
\end{array}\right.
$$

For the purpose of seeking control rate, to explore requirement of bounded joint input velocity $v$ and joint angular velocity $w$, the $\operatorname{system}\left(x_{,}^{*}, y_{c}^{\prime}, \theta_{e}\right)^{\mathrm{T}}$ should be bounded on the condition of input control and meet with the condition of $\lim _{t \rightarrow \infty}\left\|\left(x, y_{e}, \theta_{e}\right)^{\mathrm{T}}\right\|=0$ on the state of any error. On the selection of fuzzy input-output quantity, Hao Y X [7] proposed that joint input velocity $v$ affected in a smaller factor, just meeting the appropriate speed criteria, picking arm tracking can be achieved on the condition of proper joint angular velocity $w$, and speed affected mainly $x_{\mathrm{e}}$, and the selection of $x_{\mathrm{e}}$ related with the curvature, nevertheless the size of the curvature influenced directly speed, therefore fuzzy controller is designed with the following functions. First of all, to adapt fuzzy control variable of the joint velocity according to the desired path curvature, then apposed $y_{e}$ and $\theta_{e}$ control input to obtain fuzzy tracking control variable of joint angular velocity.

\section{The Picking Arm Fuzzy Control Strategy}

As can be seen from the above mention analysis, the dynamic characteristics of the picking arm trajectory planning is highly nonlinear movement with wide range speed 0.5 to $15 \mathrm{~km} / \mathrm{h}$, which features complex relationship of time domain and the system parameters widely distribution. Therefore, it is essential to establish a complete mathematical model to describe the basis of control driving requirements in order to solve the problem of stability control. Meanwhile, in view of the fuzzy logic system avoid using complex and inefficient approximate mathematical model, it can increase user experience behavior to imitate human driving anticipation and reaction behavior on the condition of not much using mathematical expression, make a stable fuzzy decision and execute driven discrimination. The fuzzy controller principle of picking-arm shown as Figure.2, the main structure consists of precise fuzzification, fuzzy control rules, fuzzy decision, fuzzy strategy. The paper design 2dimension velocity and angle velocity fuzzy controller based on trajectory tracking theory.

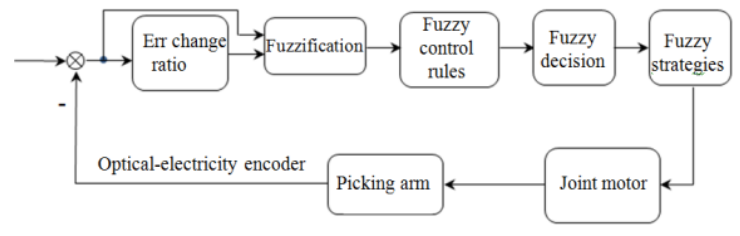

\section{Figure.2 The Fuzzy Control Principle of Picking-arm}

Generally speaking, the input language variable is respectively joint velocity error (TORE) and joint angular velocity error (TOREC). This can guarantee system reduce the overshoot and weaken the influence on start-up and acceleration vibration of picking arm.

\subsection{Non- Fuzzy Relation between Posture and the Desired Trajectory}

To indicate non-fuzzy control characteristic relationship between posture and the desired trajectory on the condition of the actual picking power transmission, obtain desired trajectory, by the literature review on trajectory depiction the arm joints drive quality center angle and 
driven time relationship diagram shown as Figure. 3 and Figure.4, so the trajectory tracking control goal is to apply the fuzzy tracking algorithm change displacement and angular velocity of the picking arm joint on complex orchard conditions, make it smooth and stable run so as to restrain its fluttering.

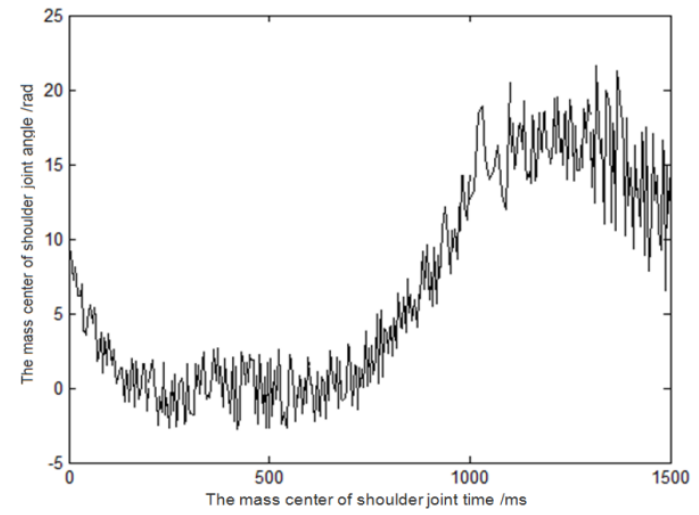

Figure.3 Driven State Diagram of the Shoulder-Joint

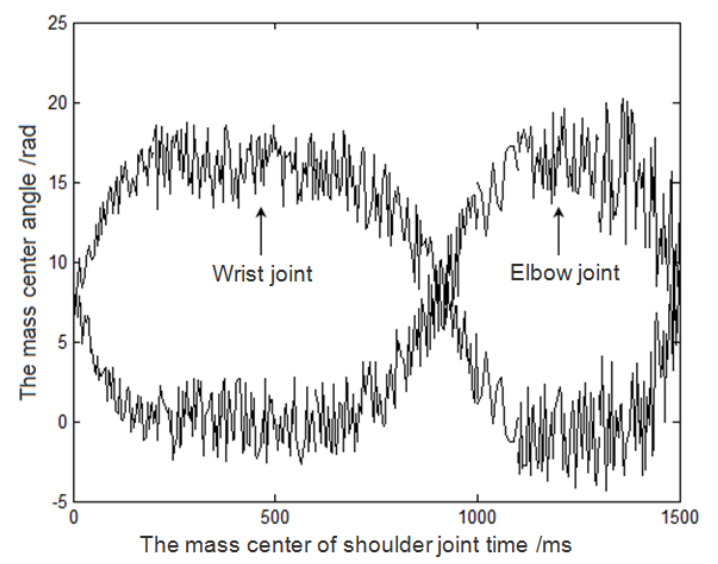

Figure.4 Driven State Diagram of Elbow-joint and Wrist-joint

Given that there is no simple mathematical relationship between each joint position and the desired trajectory, and fuzzy theory similar to the human hand picking is the intuitive choice for robotic arms picking behavior, assuming the basic domain for joint posture is stood for [$a, a]$, if the domain of error change is stood for $\mathrm{X}=[3,2.5,2,1.5,1,0.5,0,0.5,1,1.5,2.52$, $3]$, the quantitative error factors $k_{e}=6 / a$, Therefore eight language variables of err change concluded positive large (PL), positive middle (PM), positive small (PS), positive zero (PZ), and negative zero (NZ), negative small (NL), negative middle(NM) and negative large(NL).Fuzzy subset reflex hierarchical fuzzy, the corresponding control practice point out that fuzzy control is not sensitive for the fuzzy language variables shape of the membership function, but partly sensitive for scope. It can conform membership function of triangle shape $u(x), P L, P M, \ldots N L$ according to the criterion for membership function of triangle shape, Therefore evaluation of linguistic variable TORE between joint posture and expected trajectory shown as Table.1, similarly membership grade and it's evaluation of linguistic variable TOREC and $U$ can also be confirmed in their own domain. 


\section{Table.1Evaluation of Linguistic Variable TORE between Joint Posture and Expected Trajectory}

\begin{tabular}{|c|c|c|c|c|c|c|c|c|c|c|c|c|c|}
\hline \multirow{2}{*}{$\begin{array}{l}\text { TOR } \\
E\end{array}$} & \multicolumn{13}{|c|}{ TORE } \\
\hline & -3 & -2.5 & $-2 \quad-1.5$ & -1 & -0.5 & $\begin{array}{ll}0 & 0.5 \\
\end{array}$ & 1 & 1.5 & $\begin{array}{ll}2 & 2.5 \\
\end{array}$ & 3 & & & \\
\hline$N L$ & 0.8 & 0.6 & 0.3 & & & & & & & & & & \\
\hline$N M$ & & 0.3 & 1 & 0.4 & & & & & & & & & \\
\hline$N S$ & & & & 0.3 & 0.9 & & & & & & & & \\
\hline$N Z$ & & & & & & 0.3 & 0.9 & & & & & & \\
\hline$P Z$ & & & & & & & 1.0 & 0.2 & & & & & \\
\hline$P S$ & & & & & & & & 0.2 & 1.0 & 0.6 & & & \\
\hline$P M$ & & & & & & & & & & 0.6 & 1.0 & 0.1 & \\
\hline$P L$ & & & & & & & & & & & 0.2 & 0.7 & 0.9 \\
\hline
\end{tabular}

\subsection{Picking Arm Fuzzy Controller}

The fuzzy controller of picking robot arm holds 2 input variables, the angle error TORE and angular velocity error TOREC, an output variable $u$ and the 56 rules

(1)The angle error TORE: assuming fixed error $T_{g}$ and feedback error $T_{f}$ are linguistic variables TORE, the membership function shape shown as Figure.5, the center point of triangular shape changes correspondingly within [-0.03:0.01:0.03].

$$
\text { TORE }=T_{g}-T_{f}
$$

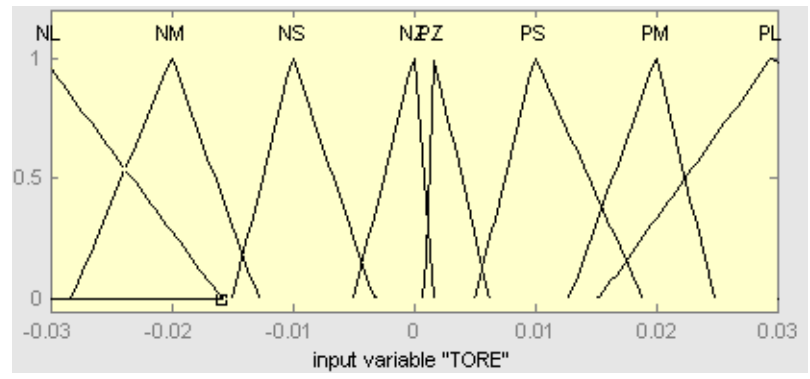

Figure.5 TORE Membership Function

(2) The angular velocity error rate of change TOREC : regarded as angular velocity differential error in discrete time, the joint angle speed error membership shown as Figure.2, the representative of the linguistic variables represent as the following

$$
\text { TOREC }=(T O R E * t * T-T O R E(t-1) * T) / \Delta t
$$

Where, differential time $t=k T(k=0,1,2 \cdots n), T$ is the sampling period, 7 triangular membership functions are defined as Figure.6, the membership center variable changed within [-0.5:0.2:0.5]. 


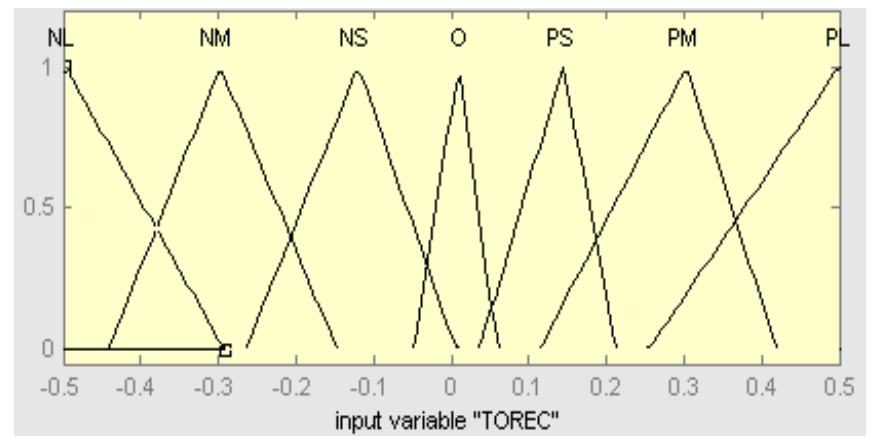

Figure.6 TOREC Membership Function

(3) Joint output variables $U$ :joint triangle shape center lies respectively $-0.2,-0.12,-0.05$, $0,0.05,0.13,0.2$,the variable shape is decided physical constraints of experiment and sensor. The command will be executed by joint servo motor in $2 \mathrm{~ms}$. Seeing that the output variable is unstable, the stabilizing treatment for improving the anti-fluttering needs to be given [10]. Therefore, the system core of picking arm fuzzy tracking control is fuzzy strategy.

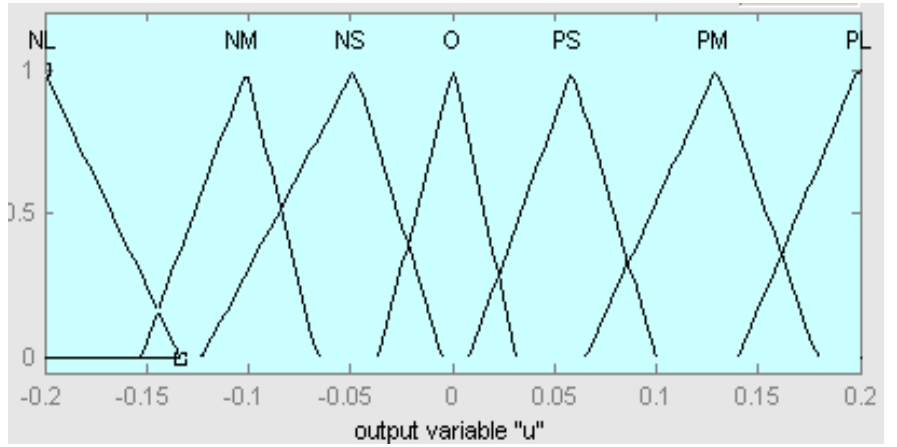

Figure.7 Joint Output Control Linguistic Variable U

\subsection{Fuzzy Control Algorithm of Picking Robot Arm}

Fuzzy control algorithm is deduced in summary from fuzzy strategy, once the fuzzy relation $R$ is confirmed in the application much picking experiments, according to description of McCarthy C. L [11] and Lee T [12], fuzzy control algorithm followed the "If TORE and TOREC then $U^{\prime \prime}$. The 56 inference language rules ${ }^{[7]}$ are proposed as following

(1) If $T O R E=N L$ and $T O R E C=N L$ then $U=P L$

(2) or If TORE $=N L$ and $T O R E C=N M$ then $U=P L$

(3) ...

(55) or If $T O R E=P M$ and $T O R E C=P L$ then $U=N L$

(56) or If $T O R E=P L$ and $T O R E C=P L$ then $U=N L$

The above 56 fuzzy control rules reflect human strategy existing in the form of statement case can be obtained. Linguistic variables are defined in fuzzy sets. The fuzzy algorithm is obtained based on the experience of rapid extraction rules as the main advantage, fuzzy rules of picking robot arm shown as Table.2 is defined 3-joints control rules statement, the corresponding joint angle can also be achieved, general fuzzy rules can be obtained according to the above-mentioned rules. 
Table.2 Fuzzy Control Rules Status of Joint

\begin{tabular}{cllllllll}
\hline \multirow{2}{*}{ TORE } & \multicolumn{7}{c}{ TOREC } \\
\cline { 2 - 8 } & $N L$ & $N M$ & $N S$ & $N Z$ & $P Z$ & $P S$ & $P M$ & $P L$ \\
\hline$N L$ & PL & PL & PL & PL & PM & PS & PS & PZ \\
$N M$ & PL & PL & PM & PM & PM & PS & PZ & PS \\
$N S$ & PL & PL & PM & PS & PS & NS & PZ & PS \\
$N Z$ & PL & PL & PM & PZ & PZ & NS & M & NL \\
$P Z$ & PL & PM & PS & NS & NS & NS & NM & NL \\
$P S$ & PL & PM & PS & NS & NM & NM & NL & M \\
$P M$ & PS & PZ & NS & NM & NL & NM & NL & M \\
$P L$ & PZ & NS & NS & NM & NL & NL & NL & NM \\
\hline
\end{tabular}

If fuzzy rules $R$ is gained, joint angle error TORE and angular velocity error TOREC can accordingly be achieved, the output of fuzzy controller can be obtained $U_{l}=T O R E_{l} \times T O R E C_{l}$, the surface view for 56 fuzzy rules can be shown as following.

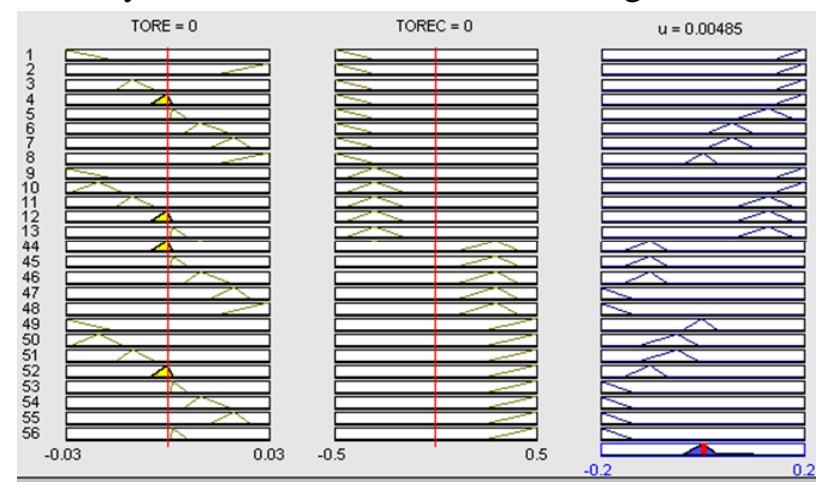

\section{Figure.8 The Surface Views of 56 Fuzzy Rules}

\subsection{Precise Fuzzy Quantity}

Fuzzy rules variable $U$ can be jointly achieved by the joint angles TORE and angular velocity error TOREC [12], triangle shape of the fuzzy membership reflect changing scope. The criterion result of fuzzy function $U$ is obtained by the summing-average method. In view of the output membership function [13] is a single variable, the variable $U$ is calculated as following.

$$
u=\frac{\sum \mu\left(x_{i}\right) x_{i}}{\sum \mu\left(x_{i}\right)}
$$

Where, $\mu\left(x_{i}\right)$ is the weighting of rule $\mathrm{i}, x_{i}$ is output variables value inferred by the fuzzy rules. The weighting values represent the global contribution from the rule library. In the process of fuzzy control, micro-controller can be obtained sampling results and transformation error TORE and error change rate TOREC, then multiply by the quantization factors $k_{T O R E}=6 / a$ and $k_{T O R E C}=6 / b$ respectively, then finally obtained control variable timely. General control array value is stored in the GT-400-SV RAM [14]. Relative linguistic variables TORE TOREC and $U$ display in 3-dimensional view. Figure.6 is 3-dimensional input and output view of fuzzy control for picking robot arm. Fuzzy control system for 
picking arm output "PM", "PS", "PL", "PO", "NZ", "NS", "NM" and "NL" is limited in the range of $[-0.2-0.2][8]$, linguistic values are inferred from each rule, generalized from fuzzy accurate program to the brief value, then output the analog signal to the joint motor, the motor therefore, drive the picking robot arm joint to meet timely the expectations picking motion.

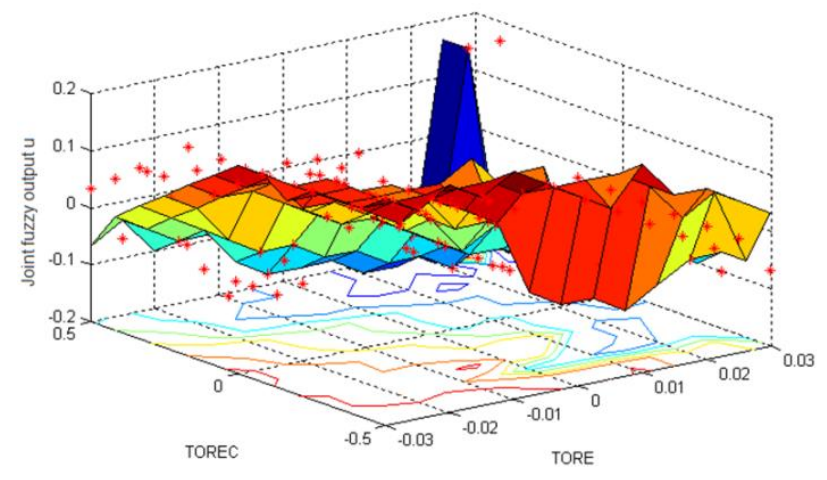

Figure.9 3-dimension View of Linguistic Variable for Picking-arm Fuzzy Controller

\section{Fuzzy tracking Algorithm Design}

As for the picking arm tracking algorithm, the two order curve tracking method should be adopted, the algorithm is looked the nearest distance point from the picking apple as the desired starting point, then approximated to the desired path in sector-by-sector and two times curve solution [15], and plan two order curve and track picking trajectory timely. Although the algorithm is relatively common to the tracking path in tracking accuracy and adaptability, and it has the virtue of good stability, it is suitable for tracking rectification. The algorithm principle can be referred to Equ.6. Assuming that we select target picking point $P(v, w)$ then the deviation vector $(x, y, \theta)$ for the picking target point can be calculated in the coordinate system, utilizing two order curve to connect the target point and the origin as the expectations following trajectory. It is used to describe the arm joint angular velocity $v$ and angular acceleration $w$ in the trajectory system

$$
\begin{aligned}
& y=\mathrm{A} x^{2}, \quad \mathrm{~A}=\frac{y_{e}}{x_{e}^{2}} \\
& \left\{\begin{array}{l}
x_{e}^{2}\left(1+4 \mathrm{~A}^{2} x^{2}\right) \\
w=\frac{2 A x^{3}}{v}
\end{array}\right.
\end{aligned}
$$

Now supposing that time $n \Delta t \leq t \leq(n+1) t$

$$
\left.x=K_{n}(t-\Delta t),\right)_{n}=\frac{a}{1+A_{n}}
$$

Through the Equ.8, approximate solution can be gained as following

$$
\left\{\begin{array}{l}
v_{n} \approx K_{n} \\
w_{n}=2 A_{n} K_{n}
\end{array}\right.
$$

In fact, we may select the appropriate speed control parameter $a$ and control cycle by calculating $v_{n}$ and $w_{n}$, then adjusted timely $t$, the picking robot arm joint angular velocity $v_{n}$ and acceleration $w_{n}$, so as to reduce quickly the deviation, and gradually approach the shortest 
distance target path from the picking apple to achieve trajectory tracking correct deviation.

\section{The Simulation and Experimental Analysis}

In order to testify the validity of the fuzzy tracking control algorithm, we design error relationship experiment of picking robot arm joints with disturbance and non-disturbance in the two aspects respectively.

(1)To embody position tracking performance on the trajectory tracking fuzzy controller, for one picking trajectory point, the robot arm joints track respectively the desired trajectory at the speed of $0.18 \mathrm{rad} / \mathrm{s}, 0.16 \mathrm{rad} / \mathrm{s}, 0.16 \mathrm{rad} / \mathrm{s}$. The simulation error curve experiment is shown as Figure.10 and Figure.11

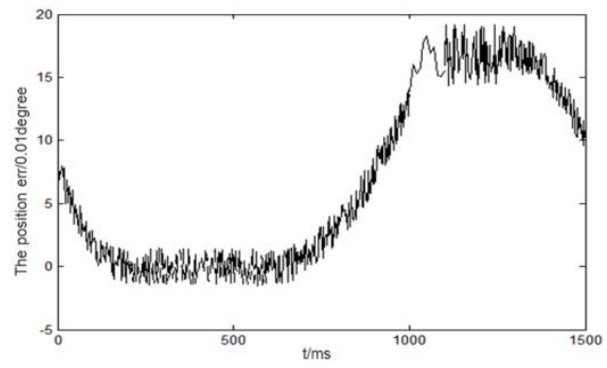

Figure.10 Shoulder Joint Position Error Curve Diagram under Fuzzy Tracking Control

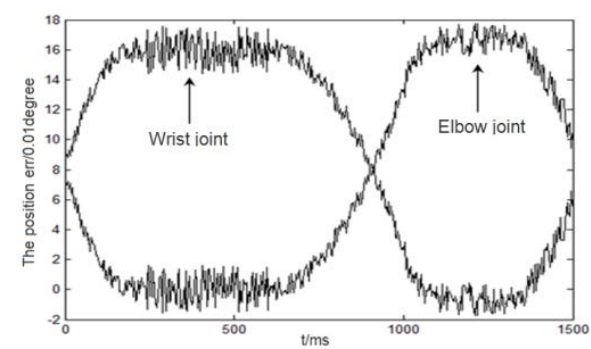

\section{Figure.11 Elbow-wrist Joint Position Error Curves Under Fuzzy Tracking Control}

(2)To embody anti-disturbance performance of the fuzzy trajectory tracking controller under the action of disturbance, exert random disturbance, which own 1.5s the duration time, maximum torque 10.8 N.M to the shoulder, elbow and wrist joints. The simulation result error disturbance curves are shown as Figure.12-Figure.14.

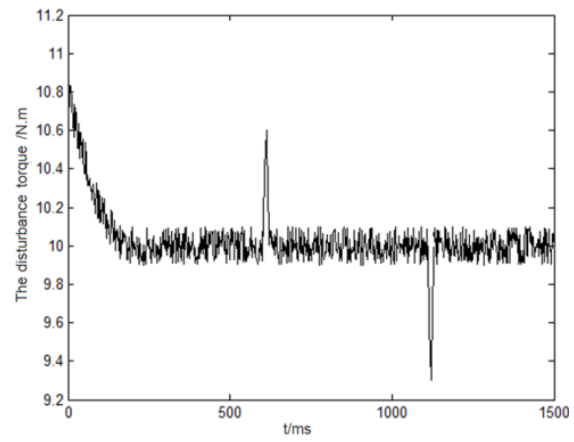

Figure.12 External Disturbance Curve 


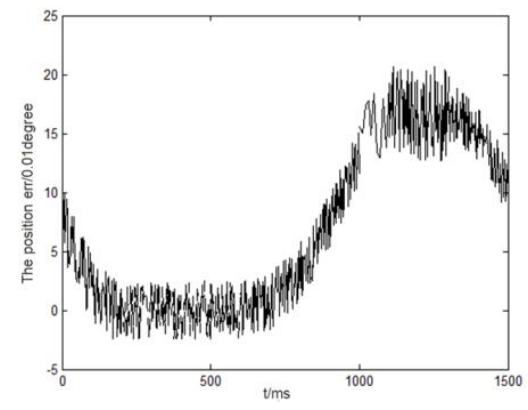

Figure.13 Shoulder Joint Position Error Curve Under the External Disturbance for Fuzzy Tracking Control

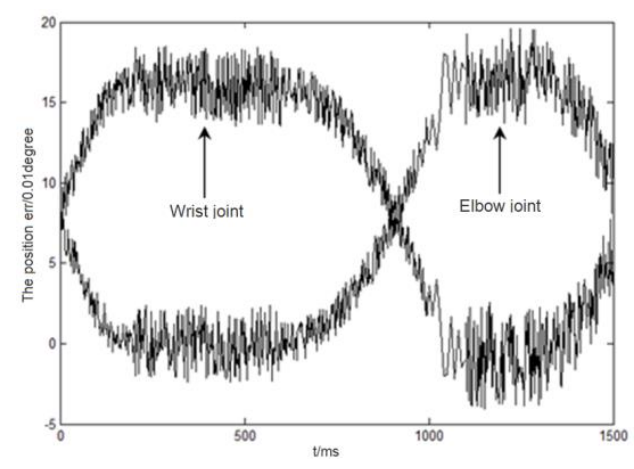

Figure.14 Elbow-wrist Joint Position Error Curves Under the External Disturbance for Fuzzy Tracking Control

Seen from Figure.13 and Figure.14, the curve between joint posture and drive wheel speed has been further improved using the fuzzy tracking control algorithm, the algorithm adjust change rate of input variables for the membership function, the picking arm joint motor make minor adjustments rather than the big control response at the motion starting or accelerating, simultaneously, the motor can reach smoothly the ideal velocity while tracking, the joint vibration is very small.

(3) As for the analysis of the joint tracking trajectory with the corresponding velocity, It can be seen from comparison between Figure.10,Figure.11 and Figure.4,Figure.4, 3-joint tracking expected trajectory by using the non-fuzzy control, angular position error is up to \pm $0.036^{\circ}$,position error consistency among the 3 joints is poor; on the condition of position tracking accuracy unchanged, the error angular position is only \pm 0.016 on the application of fuzzy tracking control, and the 3 joint own good position tracking consistency. Further analysis of the joints the inertia, the shoulder joint maximum, the elbow followed, wrist inertia minimum. Experiments show that even the premise parameters between 3 joints is inconsistent, Fuzzy tracking control of external parameters reveals good robustness without repeating adjustment parameters, the system can run in sub-optimal and optimal state.

(4)As for the analysis on the 3 joints random disturbance, 3-joints track the desired trajectory shown as Figure.13 and Figure.14. Although the error of position tracking is increasing, the largest effect on the random perturbation is shoulder joint position tracking, followed by the elbow joint. On the exertion of external disturbance torque, 3 joints can timely feedback the external force in the fuzzy tracking controller, and control rapidly the joint motor speed to compensate influence on tracking error. The 3 joints maintain 
be stable within $1.5 \mathrm{~s}$.

The experimental results show the fuzzy tracking system have something with the changing parameters of tracking object, on the external system perturbation, the trajectory tracking fuzzy controller can still make the arm joints tends to be stable in $1.5 \mathrm{~s}$, reflecting the strong robustness and stability of the tracking control system, which is conducive to restrain picking robot arm chattering.

\section{Conclusion}

Aiming at the problem of the controller stability caused by parameter variations, joint control system disturbance, modeling error, we propose a trajectory tracking fuzzy controller based on the fuzzy correction principle, which is use error to correct the output of controller. The controller can overcome the uncertainty, guaranteeing the system run closely in optimal state. Simulation results show that the trajectory tracking controller has a good adaptability for parameter variations and external disturbance, it can still maintain the arm joints keep stability in $1.5 \mathrm{~s}$ in disturbance avoiding the complicated mathematical modeling, nonlinear calculation, providing beneficial reference for the study of other similar manipulator stability.

\section{Acknowledgements}

This work is funded by Major Projects Plan of 2010 ShaanXi Tobacco Science and Techno- logy Company, Technology Innovation Foundation of Northwest A\&F University (No.QN 2013051) and Doctor Scientific Research Startup Project(No.2013BSJJ106).

Corresponding author:Zhang Jianfeng(1968-) Male,Ph.D, College of information Engineering, Northwest A\&F University, Yangling,Shaanxi. Research field: Machine vision and picking robot path planning.

\section{References}

[1] Z. MingLu, J. Xinjing, C. Zuoliang, “A new mobile robot path tracking control strategy[J]”, Journal of HeBei University of Technology, vol. 29, no. 1, (2000), pp. 71-75.

[2] W. Ahamed and C. Kambhampati, "Stable quantum filters with scattering phenomena", International Journal of Automation and Computing, vol. 5, no. 2, (2008), pp. 132-137.

[3] W. Xing, L. Peihuang and Y. Lei, "Intelligent predictive control of path of robot analysis based on vision tracking state", vol. 31, no. 4, (2009), pp. 357-364.

[4] M. Couceiro, D. Portugal, N. Gonçalves, R. Rocha, J. Luz, C. Figueiredo and G. Dias, "A methodology for detection and estimation in the analysis of golf putting”, Pattern Analysis \& Applications, (2009), pp. 1-10.

[5] M. Efe, "ADALINE based robust control in robotics: a Riemann-Liouville fractional differintegration based learning scheme", Soft Computing - A Fusion of Foundations, Methodologies and Applications, vol. 13, no. 1 (2009), pp. 23-32.

[6] C. Damianou, K. I oannides and N. Milonas, "Positioning device for MRI-guided high intensity focused ultrasound system", International Journal of Computer Assisted Radiology and Surgery, vol. 2, no. 6, (2008), pp. 335-344.

[7] Y. X. Hao and S. Agrawal, "Formation Planning and Control of UGVs with Trailers", Autonomous Robots[J], vol. 19 , no. 3, (2005), pp. 257-268.

[8] Z. H. Wang, L. B. Zhang, G. J. Bao, S. M. Qian and Q. H. Yang, "Design and control of integrated pneumatic dexterous robot finger", Journal of Central South University of Technology, vol. 18, no. 4, (2011), pp. 11051122.

[9] Y. G. Yin, S. Hosoe and Z. W. Luo, "A mixed logic dynamical modeling formulation and optimal control of intelligent robots", Optimization and Engineering[J], vol. 8, no. 3, (2007), pp. 321-330.

[10] B. Thuilot, C. Cariou, P. Martinet and M. Berducat, "Automatic Guidance of a Farm Tractor Relying on a Single CP-DGPS", Autonomous Robot[J]s, vol. 13, no. 1, (2002), pp. 53-71.

[11] C. L. McCarthy, N. H. Hancock and S. R. Raine, "Applied machine vision of plants: a review with implications for field deployment in automated farming operations”, Intelligent Service Robotics, vol. 3, no. 4, (2010), pp. 209-217. 
[12] T. Lee, S. Hudson and J. Y. Chang, "Auto-detection micro-controller-based autonomous band wrapping system for targeted pest control, " Microsystem Technologie], vol. 16, no. 1, (2010), pp. 227-238.

[13] J. Feng, F. Gao, X. Zhao, Y. Yue and R. Liu, "A new macro-micro dual drive parallel robot for chromosome dissection", Journal of mechanical science and technology, vo. 26, no. 1, (2012), pp.187-194.

[14] F. Farahmand, M. Pourazad and Z. Moussavi, "An intelligent assistive apple picking robotic manipulator", Conf Proc IEEE Eng Med Biol Soc, vol. 5, no. 3, (2005), pp. 5028-5031.

[15] C. Harper and G. Virk, "Towards the Development of International Safety Standards for Human Robot Interaction”, International Journal of Social Robotics, vol. 2, no. 3, (2010), pp. 229-234.

\section{Authors}

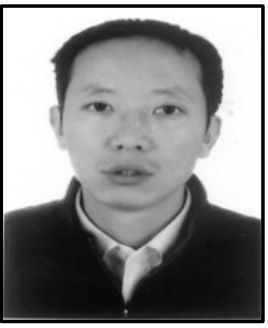

Zhang ZhiYong received the B.Eng. degree in agriculture machinery engineering in 1999, the M.Sc. degree in mechanicalelectrical integration from North-West A\&F University, YangLing, Shaanxi, China, in 2005 received the Ph.D. degrees in Agriculture mechanization from North-West A\&F University of China in 2012. His research focuses on auto control, Agricultural robot, distributed computing.

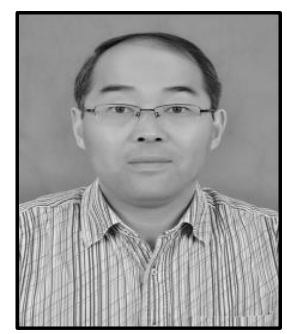

Jianfeng Zhang,Associate Professor, received the Ph.D. degree in the College of Mechanical and Electric Engineering, Northwest A\&F University, Yangling, Shaanxi, China,in 2012. His research interests mainly focus on the key technologies of intelligent control system and applications, motion control of robotic systems, and embedded systems in the application of modern agriculture.

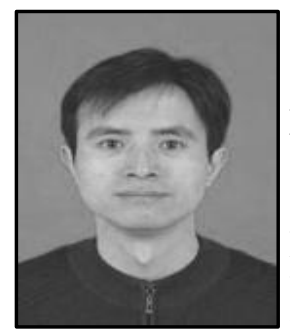

Lvwen Huang received the B.Eng. degree in automation engineering in 1999, the M.Sc. degree in signal and information processing from the Xi'an University of Technology, Xi'an, Shaanxi, China, in 2005, and Ph.D. degree in agriculture electrification and automation from College of Mechanical and Electric Engineering, Northwest Agriculture and Forestry University, Yangling, Shaanxi, China, in 2013. His research interests lie in intelligent system and image processing. He has published over 10 papers in related international conferences and journals. 